\title{
Benefit of contrast-enhanced PET/CT versus non-contrast- enhanced PET/CT relative to lesion detection, lesion characterization, and diagnostic accuracy in patients with cancer
}

Tanyaluck Thientunyakit ( $\square$ stanyalu@hotmail.com )

Mahidol University Faculty of Medicine Siriraj Hospital https://orcid.org/0000-0002-6139-7887

Phakphoom Thiravit

Mahidol University Faculty of Medicine Siriraj Hospital

Pichet Mali

Mahidol University Faculty of Medicine Siriraj Hospital

Potjanee Kanchanapiboon

Mahidol University Faculty of Medicine Siriraj Hospital

Research article

Keywords: Contrast-enhanced PET/CT, non-contrast-enhanced PET/CT, lesion detection, lesion characterization, diagnostic accuracy, cancer, [F-18]FDG

Posted Date: August 28th, 2019

DOI: https://doi.org/10.21203/rs.2.10431/v1

License: (c) (i) This work is licensed under a Creative Commons Attribution 4.0 International License. Read Full License 


\section{Abstract}

Background To investigate the benefit of contrast-enhanced PET/CT (PET/CECT) versus non-contrast-enhanced $\mathrm{PET} / \mathrm{CT}$ (PET/NCCT) relative to lesion detection, characterization, and diagnostic accuracy in cancer patients. Methods This prospective study included patients aged $>18$ years with histopathologically proven cancer who underwent [F18]FDG PET/CT at the Division of Nuclear Medicine, Department of Radiology, Mahidol University, Bangkok, Thailand during the December 2014 to November 2017 study period. PET/NCCT was performed followed by PET/CECT scan in all patients. The results of PET/NCCT, PET/CECT, and pre/post-contrast enhanced PET/CT (PET/NCCT-CECT) for each patient were interpreted by 1 nuclear medicine physician and 1 radiologist. The sensitivity, specificity, accuracy, positive predictive value (PPV), and negative predictive value (NPV) were calculated from ROC curve analysis. Results One hundred and ten patients were included. The mean age was $52.45 \pm 17.14$ years, and $52.7 \%$ were female. Lymphoma was the most common cancer diagnosis (47.3\%). No significant difference was observed between PET/CT techniques for detection rate at the primary tumor site, lymph node, or distant organ. High agreement was observed between $\mathrm{PET} / \mathrm{CT}$ techniques for lesion characterization. Lesion characterizations were not significantly correlated with age, gender, BMI, or FBS; however, lesion characterization was found to be significantly associated with primary tumor size, indication for PET/CT and lesion size. The following ranges were observed from all PET/CT techniques: sensitivity 81.5\%-85.3\%, specificity 94.4\%-95.5\%, accuracy 89.4\%-91.4\%, PLR 14.572-18.719, NLR 0.155-0.196, PPV 90.4\%-92.1\%, and NPV 88.9\%-91.3\%. Conclusions [F-18]FDG PET/CT demonstrated no significant advantage over PET/NCCT for lesion detection, lesion characterization, or diagnostic accuracy in patients with cancer. The use of intravenous contrast material should be limited to select cases in order to reduce the risk of renal toxicity and/or anaphylactic reaction, and to minimize unnecessary costs.

\section{Background}

Integrated positron emission tomography and computed tomography or PET/CT scan using [F-18] fluorodeoxyglucose ([F-18]FDG) is a well-accepted imaging technique that is used for diagnosis, staging, response assessment, recurrence detection, and follow-up among patients with suspected or definitively diagnosed cancer. PET/CT was shown to have greater diagnostic accuracy than PET or CT alone relative to primary tumor detection, lymph node metastasis, and distant metastasis. ${ }^{(1)}$ In order to obtain a diagnostically efficacious CT scan when combined with PET, the administration of intravenous contrast material may be required. The use of intravenous contrast material facilitates precise identification and differentiation of vascular and parenchymatous organ tissue from surrounding tissues. Moreover, the evaluation of lesion enhancement improves lesion detection and lesion characterization. Additionally, assessment of a lesion's relationship with adjacent vascular structures is important for surgical planning. ${ }^{(2,3)}$ Some previous studies suggested that contrast-enhanced PET/CT improves the detection and characterization of liver lesions in patients with colorectal cancer ${ }^{(2)}$, recurrent rectal cancer ${ }^{(4)}$, ovarian cancer ${ }^{(5)}$, and lung cancer. ${ }^{(6)}$ In contrast, other studies found no significant difference between non-contrast-enhanced CT and contrast-enhanced CT integrated with PET in patients with malignant lymphoma ${ }^{(7)}$, and in patients with head and neck cancer. ${ }^{(8,9)}$ Importantly, intravenous contrast material should be used only when the potential benefits outweigh the potential risks since contrast material can cause renal toxicity and/or anaphylactic reaction in some patients. Some authors recommend against routine intravenous contrast administration for PET/CT, but they agree that it may be selectively used in patients with earlystage head and neck cancer that requires meticulous anatomic and topographic data to plan the operation, and in patients with advanced-stage cancer for assessing vascular invasion. ${ }^{(9)}$ No consensus has yet been reached regarding if, how, and when intravenous contrast material should be used in PET/CT study.

Accordingly, the aim of this study was to investigate the benefit of contrast-enhanced PET/CT versus non-contrastenhanced PET/CT relative to lesion detection, characterization, and diagnostic accuracy in patients with cancer. 


\section{Methods}

A flow diagram describing patient enrollment and the study protocol is shown in Figure 1.

\section{Patients}

This prospective study was conducted in patients aged $>18$ years with histopathologically proven cancers who underwent [F18]FDG whole body PET/CT scan at the Division of Nuclear Medicine, Department of Radiology, Faculty of Medicine Siriraj Hospital, Mahidol University, Bangkok, Thailand during the 1 December 2014 to 30 November 2017 study period. Patients with severe renal dysfunction (glomerular filtration rate $[G F R]<20 \mathrm{ml} /$ minute; $\mathrm{n}=3$ ), contraindications for contrast material ( $\mathrm{n}=2$ ), and marked hyperglycemia (fasting blood sugar [FBS] >200 mg/dl; $n=2$ ) were excluded.

All PET/CT studies were performed at least 4 weeks after biopsy or surgery, 6 weeks after chemotherapy, 12 weeks after radiotherapy, and 2 weeks after granulocyte-colony stimulating factor (GCSF) treatment to prevent falsepositive results due to inflammatory changes. Clinical, histopathological, radiological follow-up, and other correlative investigation were used as reference standards to evaluate the diagnostic accuracy of PET/CT.

\section{PET/CT protocol}

All data were acquired using an integrated PET/CT system (Discovery ${ }^{\circledR}$; GE Healthcare, Milwaukee, WI, USA) that integrates a 16-detector row CT scanner with a lutetium oxyorthosilicate (LSO)-based PET scanner. Patients fasted at least 6 hours prior to [F-18]FDG administration, and blood glucose level was checked using a glucose meter (Stat Strip ${ }^{\circledR}$; Nova Biomedical, Waltham, MA, USA) just before [F-18]FDG administration. Tracer injection was performed only in patients whose blood glucose level was less than $150 \mathrm{mg} / \mathrm{dL}$. Whole-body emission images were obtained 60 minutes after intravenous administration of [F-18]FDG (0.14-0.2 mCi/ kg), with an average injected dose of 381.19 $\pm 76.4 \mathrm{MBq}$ $(10.3 \pm 2.0 \mathrm{mCi})$.

In all patients, $2 \mathrm{ml} / \mathrm{kg}$ of non-ionic contrast material using either lopamidol (lopamiro ${ }^{\circledR}$; Bracco Imaging, Milano, Italy) or lohexol (Omnipaque ${ }^{\circledR}$; GE Healthcare, Milwaukee, WI, USA) or loversol (Optiray ${ }^{\circledR}$; Guerbet, Cedex, France) or lodixanol (Visipaque ${ }^{\circledR}$, GE Healthcare, Milwaukee, WI, USA) was intravenously injected. Whole body CT (30-300 mAs using Automatic Exposure Control (AEC) and smart mA, 14.0 noise index, $120 \mathrm{kVp}$, and helical thickness of $2.5 \mathrm{~mm}$ collimation) was performed immediately before and after contrast injection. Contrast-enhanced CT scan was performed after contrast injection using the appropriate delay time (40 seconds for lesion at head/neck/chest, and 70 seconds for lesion at abdomen). PET data was acquired in 3-D mode, 3 minutes per bed position, and reconstruction was performed using an ordered subset expectation maximization algorithm (3D Iterative [VUE Point]).

\section{Image interpretation}

The reconstructed, attenuation-corrected images of all PET/CT datasets were reviewed by one board-certified nuclear medicine physician (14-years' experience) and one diagnostic radiologist (11-years' experience), both of whom were blinded to patient clinical information, using an AW Workstation (GE Healthcare, Milwaukee, WI, USA). Images without attenuation correction were available for evaluation in cases with suspicious artifacts. For non-contrast PET/CT, a lesion was defined as either a focus of increased [F-18]FDG uptake compared with background, or as morphologic change with features that increase suspicion of the presence of a tumor. For contrast-enhanced PET/CT, abnormal enhancement was added to the criteria adopted for non-contrast PET/CT (e.g., enhancement $>15 \mathrm{HU}$ in

pulmonary nodule sized $>8 \mathrm{~mm})$. $^{(10-13)}$ Lymph node metastasis was considered in lymph nodes with FDG avidity and when their shortest axial diameter was $>11 \mathrm{~mm}$ in the jugulodigastric region and $>10 \mathrm{~mm}$ in the 
cervical/abdominal/pelvic region ( $>5 \mathrm{~mm}$ in rectal cancer), or if irregular border or central necrosis was evident, or if there was a cluster of three or more lymph nodes of borderline size. ${ }^{(14-16)}$ Distant metastasis was defined as a focally increased [F-18]FDG activity compared with background, with associated soft tissue mass outside of the primary lesion or bony destruction. Equivocal lesion detected by PET or CT that failed to satisfy any of the aforementioned diagnostic criteria was designated as an indeterminate lesion.

\section{Outcome assessment}

All whole body PET/CT studies were assessed using an 8-point scale, as follows; $\mathbf{0}=$ no abnormality detected; $\mathbf{1}=$ focal FDG uptake without CT abnormality; $\mathbf{2}$ = focal FDG uptake with CT abnormality, favoring benign; $\mathbf{3}=$ focal FDG uptake with CT abnormality, favoring malignant; $\mathbf{4}=$ CT abnormality without FDG avidity, favoring benign; $\mathbf{5}=\mathrm{CT}$ abnormality without FDG avidity, favoring malignant; $\mathbf{6}=$ focal FDG uptake with CT abnormality, indeterminate; and, $7=$ CT abnormality without FDG avidity, indeterminate. These lesions were then classified as indeterminate (scores 1,6 , or 7 ); definite benign lesion (scores 2 or 4); or, definite malignant lesion (scores 3 or 5). Both observers reviewed PET/NCCT first, followed by PET/CECT and PET/NCCT-CECT, with at least a 3-week interval between each of the 3 sets to prevent recall bias.

Study outcome was assessed by comparing total number, characterization scores (0-7), and diagnostic confidence (determinate $v s$. indeterminate) for all lesions detected by the three PET/CT techniques. Both patient-based and lesionbased data were collected and analyzed. To compare diagnostic performance, we compared the interpretation obtained from PET/CT studies with the corresponding results from intra-operative findings, pathologic study, or change in imaging findings during a minimum follow-up period of 6 months. Among patients with no intraoperative findings, lesions that decreased in size or that remain unchanged without receiving any further treatment were considered benign, while progressive lesions were considered to be malignant.

\section{Statistical analysis and sample size calculation}

All data were analyzed using the statistical software package PASW Statistics for Windows, Version 18.0 (SPSS, Inc., Chicago, IL, USA) and MedCal version 18.2.1 (MedCalc Software bvba, Ostend, Belgium; http://www.medcalc.org; 2018). Categorical variables were analyzed using chi-square test, and continuous variables were tested using one-way analysis of variance (ANOVA) with post hoc analysis. McNemar's test was used to determine the statistical significance of differences in lesion detection accuracy by PET/NCCT, PET/CECT, and PET/NCCT-CECT. Kappa statistic was used to analyze agreement of interpretation between PET/CT techniques. For all tests, a $p$-value less than 0.05 was considered statistically significant. Receiver operating characteristic (ROC) curve analysis was performed to assess diagnostic performance relative to the results obtained from intra-operative finding, pathological study, or change in imaging findings from each technique. The sensitivity, specificity, accuracy, likelihood ratio, positive predictive value (PPV), and negative predictive value (NPV) of all techniques were determined. Net reclassification improvement (NRI) of PET/CECT as compared to PET/NCCT was also calculated, with malignancy from final diagnosis being considered an event.

The sample size for this study was calculated using data from a study by Cantwell, et al. ${ }^{(2)}$ Using a detection rate of liver lesion by non-contrast enhanced PET/CT in patients with colorectal cancer of $70 \%$, a significance level of 0.05 , and a power of $80 \%$, the calculated sample size was 33 . To cover the three most common types of cancer for which PET/CT study is requested and to compensate for a $10 \%$ loss of data for any reason, the calculated minimum sample size was 110 patients.

\section{Results}


There were 110 cancer patients included. The types of cancer that were presented included lymphoma, lung cancer, gastrointestinal and hepatobiliary tract cancer, genitourinary cancer, and other cancers (5 breast cancer, 3 sarcoma, 2 melanoma, 2 metastatic cancer of unknown primary, and 1 thyroid cancer). The mean age of patients was $52.45 \pm 17.14$ years, and $52.7 \%$ were female. Approximately one-third of patients were indicated for PET/CT to monitor response during treatment - with or without baseline study. Detailed demographic and clinical data of the study population are shown in Table 1.

\section{Lesion detection and characterization}

No significant difference was observed between the PET/CT technique used and the total/individual lesion score at the primary tumor site, lymph node, or distant organ. The details of lesions detected by each PET/CT technique are summarized in Table 2. Indeterminate lesions (scores 1, 6, and 7) were found at the primary site in 7/53 (13.2\%), 6/55 (10.9\%), and 6/56 (10.7\%) of cases; at lymph node in 8/82 (9.8\%), 6/84 (7.1\%), and 6/84 (7.1\%) of cases; and, at distant metastatic site in 33/93 (35.5\%), 33/95 (34.7\%), and 31/95 (32.6\%) of cases with PET/NCCT, PET/CECT, and PET/NCCT-CECT, respectively. No significant difference in indeterminate lesions was found among PET/CT techniques. There was very high agreement for diagnostic characterization of these lesions among PET/CT techniques $(p>0.05)$ relative to both score and diagnostic confidence (determinate vs. indeterminate) (Table 3 ).

We also evaluated correlation between potential factors and the possibility of indeterminate lesions detected by each $\mathrm{PET} / \mathrm{CT}$ technique, as shown in Table 4. Overall, there was no statistically significant difference in diagnostic confidence between any PET/CT technique and gender, primary tumor type, or indication for PET/CT at primary tumor site, lymph node, or distant metastatic site. However, statistically significant differences in diagnostic confidence were observed at distant metastatic sites relative to primary tumor type (mostly in GU and GI systems with $p$-values of 0.026 , 0.017, and 0.003 in PET/NCCT, PET/CECT, and PET/NCCT-CECT, respectively), and indication for PET/CT (mostly in CUP with $p$-values of 0.07 and 0.025 in PET/CECT and PET/NCCT-CECT, respectively).

From 371 total detectable lesions, the most common location was thoracic lymph node $(n=76)$, followed by lung $(n=59)$, bone $(n=47)$, liver $(n=44)$, and abdominal lymph node $(n=31)$. The average size of all 291 measurable lesions was $1.73 \pm 1.68 \mathrm{~cm}$. From ROC curve analysis, the best cut-off for detectable lesion size was $1.25 \mathrm{~cm}$, with a sensitivity and specificity of $71.1 \%$ and $71.2 \%$, respectively. Interestingly, there were significant differences in lesion size between definite and indeterminate lesions in some techniques, but in the opposite ways. Specifically, inderminate lesions tended to be found more often in larger lesions at the primary site, but in smaller lesions at distant metastatic sites.

\section{Diagnostic performance}

There were 17 patients (15.5\%, 48 lesions) who did not follow-up at our center, so their final outcome data were not available. The sensitivity, specificity, accuracy, PPV, and NPV of PET/CT techniques in the remaining 93 patients were evaluated against the corresponding results of intra-operative finding in 14 patients (17 lesions; 16 malignant, 1 benign). In the other 79 patients, 97 lesions were considered to be malignant based on observed disease progression, and 209 lesions were considered to be benign due to no change or decrease in lesion size during the mean follow-up of 30 months. The following ranges were observed from all PET/CT techniques: sensitivity $81.5 \%-85.3 \%$, specificity 94.4\%-95.5\%, accuracy 89.4\%-91.4\%, PLR 14.572-18.719, NLR 0.155-0.196, PPV 90.4\%-92.1\%, and NPV 88.9\%-91.3\% (Figure 2). The AUC obtained from ROC curve analysis was $0.9,0.902$, and 0.88 for PET/NCCT, PET/CECT, and $\mathrm{PET} /$ NCCT-CECT, respectively. The indeterminate lesions (81 lesions in PET/NCCT, 65 lesions in PET/CECT and $\mathrm{PET} /$ NCCT-CECT) were excluded from diagnostic performance evaluation, but they are discussed further in the

Discussion section. Based on the likelihood of malignancy per each lesion score (Table 5), lesion with positive FDG 
uptake and characteristics indicative of malignancy from CT findings (score 3) showed the highest likelihood of malignancy in all techniques, followed by lesion with evidence of malignancy by CT without FDG uptake (score 5). Other lesions without suggestive evidence of malignancy either by PET together with CT or CT alone showed relatively low likelihood of malignancy.

Of the other 252 lesions with definite impression by both PET/NCCT and PET/CECT, there was no lesion that was identified as benign in PET/NCCT that was changed to malignant by PET/CECT (or vice versa) regardless of final diagnosis group. Therefore, the calculated $\mathrm{NRI}_{\mathrm{e}}, \mathrm{NRI}_{\text {ne, }}$ and sum NRI were all 0 (Table 6).

\section{Adverse reaction from contrast material}

Two patients developed skin rash within 24 hours after intravenous contrast injection for a prevalence of adverse reaction of $1.8 \%$. Both events were mild in severity, and no active treatment was required. No serious adverse events were observed during this study.

\section{Discussion}

In contrast to the added beneficial effect of intravenous contrast material found and reported in some studies ${ }^{(2-6)}$, we found no significantly added value from the use of intravenous contrast material relative to diagnostic confidence or diagnostic performance of [F-18]FDG PET/CT study using CECT alone or combined NCCT/CECT. The detection rate, diagnostic confidence as defined by percentage of indeterminate lesions, and diagnostic performance of PET/CECT and PET/NCCT-CECT were almost identical. This can be explained by the fact that the main component of results interpretation on PET/NCCT-CECT is mainly based on contrast-enhanced CT image. Thus, dual CT scans provide no additional benefit; however, they expose the patient to added and unnecessary radiation, even though only minimal exposure from the low-dose CT used in PET/CT is claimed. Similar results were also observed in previous studies in patients with lymphoma ${ }^{(7)}$, and head and neck cancer. ${ }^{(8-9)}$ Despite the reported added value of contrast material in PET/CT study that has been reported in cancers of the head and neck, abdominal and pelvic regions, and possibly in tumors with mild or no [F-18]FDG avidity $(2,4,5,6,8)$, our results failed to show statistically significant added value of contrast-enhanced CT regardless of the region of the detectable lesions. However, there were 16 indeterminate lesions from PET/NCCT that were definitely classified by PET/CECT, and 5 of those were found to be malignant in the final diagnosis. Most of those lesions were small intra-abdominal lesions with faint FDG activity that were easier to identify and diagnose via the use of contrast enhancement.

There was no significant correlation between incidence of indeterminate lesion at the primary site or lymph node and gender, primary cancer type, or indication for PET/CT. However, significant correlation was found between indeterminate metastatic lesions with primary cancer type and indication for PET/CT, particularly the PET/CECT and PET/NCCT-CECT techniques. The highest incidence of indeterminate metastatic lesions was found in approximately half of genitourinary cancer, gastrointestinal tract cancer, and hepatobiliary tract cancer, and in approximately two-thirds of patients indicated for PET/CT due to cancer of unknown primary (CUP) and for diagnosis of suspicious lesion(s). Most indeterminate lesions (71 in PET/NCCT and 55 in PET/CECT) demonstrated mild FDG uptake. As a result, the findings from CT did not fulfil the criteria for malignancy. Even though the use of contrast material seemed to lower the number of indeterminate lesions, the difference between PET/CT techniques was not statistically significant. Considering the highest likelihood of malignancy that can be obtained from lesions with positive findings from both FDG PET and CT, while the lowest likelihood in lesions detected by CT only without FDG uptake, unless strong evidence of malignancy from CT, the significance of these additional non-FDG avid lesions might be relatively low. In additional lesions detected by CT without FDG avidity, a final diagnosis of malignancy was found in 11/135 (8.1\%) vs. 11/125 (8.8\%), 3/4 (75\%) vs. $3 / 4(75.0 \%)$. and $5 / 24(20.8 \%)$ vs. $5 / 34(14.7 \%)$ of lesions classified as benign, malignant, and indeterminate by 
NCCT and CECT, respectively. All of these results show no significant incremental advantage of intravenous material in PET/CT study. In addition, the unnecessary use of contrast material should be avoided due to its potentially serious side effects. The incidence rate of side effects in this study was a low $1.8 \%$ due to our strict adherence to settingspecific guidelines. Moreover, the cost of contrast material is approximately 100 USD per patient, which makes it a high unnecessary expense in cases that will not benefit from contrast enhancement. Taken together, our data do not support the routine use of contrast material in whole body PET/CT study.

We admit that this study has some limitations. First, this was a single-center study with a relatively small sample size compared to previous publications. [F-18]FDG PET/CT study in Thailand has limited indications for reimbursement (currently only in colorectal cancer and non-small cell lung cancer), so this technique is still underutilized. This explains why the sample size is so small and why the study period is so long. Second, most of the enrolled PET/CT studies were performed post-treatment, which may affect the detection rate of malignant diseases. The prevalence of malignancy was only $35 \%$ among all detectable lesions. Third, only a small number of case $(n=14,12.7 \%)$ had pathologically proven lesions detected by PET/CT, particularly among lesions identified as benign or indeterminate. However, with the mean follow-up of approximately 2 years, lesions that decreased in size or that remain unchanged without receiving any further treatment were considered benign, while progressive lesions were considered to be malignant. Fourth and last, contrast-enhanced PET/CT in this study was performed in single-phase manner, so our results may not be generalizable to multi-phase contrast-enhanced PET/CT, which was previously suggested by some authors. ${ }^{(17)}$

In contrast to other reports that studied in specific types of cancer, the strengths of this study are its prospective design and the fact that we studied different types of cancer, which increases the generalizability of our findings. However, further investigation in those cancers with a small number of cases, as well as in other indications, is needed to confirm the results of this study.

\section{Conclusions}

The results of this prospective study revealed no significant advantage of [F-18]FDG PET/CT over PET/NCCT for lesion detection, lesion characterization, or diagnostic accuracy in patients with cancer. Although the rate of adverse events was extremely low, the use of intravenous contrast material should be limited to select cases in order to reduce the risk of renal toxicity and/or anaphylactic reaction, and to minimize unnecessary costs.

\section{Abbreviations}

FBS: fasting blood sugar

FDG: Fluorodeoxyglucose

GFR: glomerular filtration rate

LSO: lutetium oxyorthosilicate

NRI: Net reclassification improvement

NPV: negative predictive value

PET/CT: positron emission tomography/computed tomography

PET/CECT: contrast-enhanced PET/CT 
PET/NCCT: non-contrast-enhanced PET/CT

PET/NCCT-CECT: contrast-enhanced and non-contrast-enhanced PET/CT

PPV: positive predictive value

\section{Declarations}

\section{Acknowledgements}

The authors gratefully acknowledge Dr. Akarin Nimmannit for being our project facilitator, Miss Pimrapat Tengtrakulchareon for assistance with developing our research methodology, Miss Dollaporn Polyeam for assistance with data collection and analysis, and Dr. Chulaluk Komoltri and Miss Nerisa Thornsri for assistance with statistical analysis.

\section{Funding}

This study was supported by a grant from the Siriraj Research Development Fund (Managed by Routine to Research Unit: R2R), Faculty of Medicine Siriraj Hospital, Mahidol University (grant no. 14RD03017/074/14), and by a Chalermprakiat Grant from the Faculty of Medicine Siriraj Hospital, Mahidol University to TT, PT, and PK.

\section{Availability of Data and Materials}

Please contact author for data requests.

\section{Authors contribution}

TT contributed in concepts, design, literature search, clinical studies, images interpretation, data analysis, manuscript preparation and guarantor, PT contributed in design, literature search, clinical studies, images interpretation, PM and PK contributed in clinical studies, data acquisition and reconstruction. All authors have read and approved the manuscript for publication.

\section{Ethical Approval and Consent to participate}

This study was approved by the Siriraj Institutional Review Board (SIRB) (COA no. Si413/2014), and all patients provided written consent before enrollment into the study.

\section{Consent for publication}

Not applicable.

\section{Competing interest}

All authors declare no personal or professional conflicts of interest, and no financial support from the companies that produce and/or distribute the drugs, devices, or materials described in this report.

\section{References}

1. El-Galaly TC, Gormsen LC, Hutchings M. PET/CT for Staging; Past, Present, and Future. Semin Nucl Med. 2018;48:4-16. 
2. Cantwell CP, Setty BN, Holalkere N, Sahani DV, Fischman AJ, Blake MA. Liver lesion detection and characterization in patients with colorectal cancer: a comparison of low radiation dose non-enhanced PET/CT, contrast-enhanced PET/CT, and liver MRI. J Comput Assist Tomogr. 2008; 32:738-44.

3. Antoch G, Freudenberg LS, Beyer T, Bockisch A and Debatin JF. To Enhance or Not to Enhance? 18F-FDG and CT Contrast Agents in Dual-Modality 18F-FDG PET/CT. J Nucl Med. 2004;45:56S-65S.

4. Soyka JD, Veit-Haibach P, Strobel K, Breitenstein S, Tschopp A, Mende KA, et al. Staging pathways in recurrent colorectal carcinoma: is contrast-enhanced 18F-FDG PET/CT the diagnostic tool of choice? J Nucl Med. 2008; 49:354-61.

5. Kitajima K, Murakami K, Yamasaki E, Domeki Y, Kaji Y, Fukasawa I, et al. Performance of integrated FDG$\mathrm{PET} /$ contrast-enhanced CT in the diagnosis of recurrent ovarian cancer: comparison with integrated FDG-PET/noncontrast-enhanced CT and enhanced CT. Eur J Nucl Med Mol Imaging. 2008; 35:1439-48.

6. Behrendt FF, Temur Y, Verburg FA, Palmowski M, Krohn T, Pietsch H, et al. PET/CT in lung cancer: Influence of contrast medium on quantitative and clinical assessment. Eur Radiol. 2012; 22:2458-64.

7. Rodríguez-Vigil1 B, G'omez-Le'on N, Pinilla1 I, Hern'andez-Maraver D, Coya J, Martín-Curto L, et al. PET/CT in lymphoma: prospective study of enhanced full-dose PET/CT versus unenhanced low-dose PET/CT. J Nucl Med. 2006; 47:1643-48.

8. Goerres GW, Schuknecht B, Schmid DT, Stoeckli SJ, Hany TF. Positron emission tomography/computed tomography for staging and restaging of head and neck cancer: comparison with positron emission tomography read together with contrast-enhanced computed tomography. Clin Imag. 2008; 32:431-7.

9. Yoshida K, Suzuki A, Nagashima T, Lee J, Horiuchi C, Tsukuda M, et al. Staging primary head and neck cancers with (18)F-FDG PET/CT: is intravenous contrast administration really necessary? Eur J Nucl Med Mol Imaging. 2009; 36:1417-24.

10. Erasmus JJ, Connolly JE, McAdams HP, Roggli VL. Solitary pulmonary nodules. I. Morphologic evaluation for differentiation of benign and malignant lesions. Radiographics. 2000; 20:43-58.

11. Erasmus JJ, Connolly JE, McAdams HP, Roggli VL. Solitary pulmonary nodules. II. Evaluation of indeterminate nodule. Radiographics. 2000; 20:59-66.

12. Swensen SJ, Viggiano RW, Midthun DE, et al. Lung nodule enhancement at CT: multicenter study. Radiology. 2000; 214:73-80.

13. Austin JH, Garg K, Aberle D, Yankelevitz D, Kuriyama K, Lee HJ, et al. Radiologic implications of the 2011 classification of adenocarcinoma of the lung. Radiology. 2013; 266:62-71.

14. Ng SH, Yen TC, Laio CL, Chang JT, Chan SC, Ko SF, et al. 18F-FDG PET and CT/MRI in oral cavity squamous cell carcinoma: a prospective study of 124 patients with histologic correlation. J Nucl Med. 2005; 46:1136-43.

15. UyBico SJ, Wu CC, Suh RD, Le NH, Brown K, Krishnam MS. Lung cancer staging essentials: the new TNM staging system and potential imaging pitfalls. Radiographics. 2010; 30:1163-81.

16. Kaur H, Choi H, You YN, Rauch GM, Jensen CT, Hou P, et al. MR imaging for preoperative evaluation of primary rectal cancer: practical considerations. Radiographics. 2012;32:389-409

17. Morbelli S, Conzi R, Campus C, Cittadini G, Bossert I, Massollo M, et al. Contrast-enhanced [18 F] fluorodeoxyglucose-positron emission tomography/computed tomography in clinical oncology: tumor-, site-, and question-based comparison with standard positron emission tomography/computed tomography. Cancer Imag. 2014;14:1-10.

\section{Tables}

Page 9/16 
Table 1. Demographic and clinical data of the 110 included cancer patients

\begin{tabular}{|l|l|c|}
\hline \multicolumn{1}{|c|}{ Characteristics } & & \\
\hline Age (year) & (mean \pm SD) & $52.45 \pm 17.14$ \\
\hline Gender & Female, n (\%) & $58(52.7 \%)$ \\
& Male, n (\%) & $52(47.3 \%)$ \\
\hline Primary cancer type & Lymphoma, n (\%) & $52(47.3 \%)$ \\
& Lung, n (\%) & $19(17.3 \%)$ \\
& GI \& HB tract, n (\%) & $19(17.3 \%)$ \\
& Genitourinary, n (\%) & $8(7.3 \%)$ \\
& Other, n (\%) & $12(10.8 \%)$ \\
\hline Indication for PET/CT & Monitoring treatment response, n (\%) & $42(38.2 \%)$ \\
& Suspected recurrence, n (\%) & $26(23.6 \%)$ \\
& Re-staging, n (\%) & $20(18.2 \%)$ \\
& Initial staging, n (\%) & $13(11.8 \%)$ \\
& Diagnosis for suspicious lesion, n (\%) & $5(4.5 \%)$ \\
& Unknown primary tumor, n (\%) & $4(3.6 \%)$ \\
\hline BMI (kg/m ${ }^{2}$ ) & (mean \pm SD) & $23.3 \pm 3.55$ \\
\hline Fasting blood glucose level (mg/dl) on the day of PET/CT & (mean \pm SD) & $94.25 \pm 14.71$ \\
\hline Contrast material & Iohexol, n (\%) & $66(60.0 \%)$ \\
& Ioversol, n (\%) & $22(20.0 \%)$ \\
& Iopamidol, n (\%) & $11(10.0 \%)$ \\
& Iodixanol, n (\%) & $11(10.0 \%)$ \\
\hline
\end{tabular}

GI, gastrointestinal tract; HB, hepatobiliary tract; BMI, body mass index

Table 2. PET/CT findings relative to primary tumor $(\mathrm{P})$, lymph node metastasis $(\mathrm{N})$, and distant metastasis $(\mathrm{M})$ 


\begin{tabular}{|c|c|c|c|c|c|c|c|c|}
\hline \multirow[t]{2}{*}{ Lesion } & \multirow[t]{2}{*}{ Score } & \multicolumn{2}{|c|}{$\begin{array}{c}\text { PET/NCCT } \\
\text { N (\%) }\end{array}$} & \multicolumn{2}{|c|}{$\begin{array}{c}\text { PET/CECT } \\
\text { N (\%) }\end{array}$} & \multicolumn{2}{|c|}{$\begin{array}{c}\text { PET/NCCT-CET } \\
\text { N (\%) } \\
\end{array}$} & \multirow[b]{2}{*}{ p value } \\
\hline & & Patient-based & Lesion-based & Patient-based & Lesion-based & Patient-based & Lesion-based & \\
\hline \multirow{9}{*}{$\begin{array}{l}\text { Primary } \\
\text { tumor }\end{array}$} & 0 & 57 (51.8\%) & - & 55 (50\%) & - & $54(49.1 \%)$ & - & \multirow{9}{*}{$\begin{array}{c}0.50^{* *} \\
1.00^{* * *} \\
0.25^{* * * *}\end{array}$} \\
\hline & & $2(1.8 \%)$ & $2(2.5 \%)$ & $1(0.9 \%)$ & $1(1.2 \%)$ & $1(0.9 \%)$ & $1(1.1 \%)$ & \\
\hline & 1 & $5(4.5 \%)$ & $5(6.3 \%)$ & 7 (6.4\%) & 7 (8.2\%) & 7 (6.4\%) & 8 (9.1\%) & \\
\hline & 2 & 30 (27\%) & 49 (61\%) & 30 (27.3\%) & 49 (57.6\%) & 30 (27.3\%) & 49 (55.7\%) & \\
\hline & 3 & 14 (13\%) & 19 (24\%) & 15 (13.6\%) & 22 (25.9\%) & 17 (15\%) & 25 (28\%) & \\
\hline & 4 & $0(0 \%)$ & $0(0 \%)$ & $0(0 \%)$ & $0(0 \%)$ & $0(0 \%)$ & $0(0 \%)$ & \\
\hline & 5 & $4(3.6 \%)$ & 4 (5\%) & $4(3.6 \%)$ & $4(4.7 \%)$ & $4(3.6 \%)$ & $4(4.5 \%)$ & \\
\hline & 6 & $1(0.9 \%)$ & 1 (1.3\%) & $1(0.9 \%)$ & $2(2.4 \%)$ & $1(0.9 \%)$ & 1 (1.1\%) & \\
\hline & 7 & & & & & & & \\
\hline \multirow{10}{*}{$\begin{array}{l}\text { Lymph } \\
\text { node }\end{array}$} & 0 & 28 (25\%) & - & 27 (25\%) & - & 27 (25\%) & - & \multirow{10}{*}{$\begin{array}{c}1.00^{* *} \\
1.00^{* * *} \\
1.00^{* * * *}\end{array}$} \\
\hline & 1 & $4(3.6 \%)$ & $4(1.2 \%)$ & $1(0.9 \%)$ & $1(0.3 \%)$ & $1(0.9 \%)$ & $1(0.3 \%)$ & \\
\hline & 2 & $6(5.4 \%)$ & $8(2.3 \%)$ & $8(7.3 \%)$ & $11(3.2 \%)$ & $8(7.3 \%)$ & 11 (3.1\%) & \\
\hline & 3 & 34 (30.9\%) & 162 (47.4\%) & 34 (30.9\%) & 162 (46.6\%) & 34 (30.9\%) & 162 (46.4\%) & \\
\hline & & & 139 (40.8\%) & & 148 (42.5\%) & & 151 (43.3\%) & \\
\hline & 4 & 45 (40.9\%) & $0(0 \%)$ & 46 (41.8\%) & $0(0 \%)$ & 46 (41.8\%) & $0(0 \%)$ & \\
\hline & & & 25 (7.3\%) & & 24 (6.9\%) & & $23(6.6 \%)$ & \\
\hline & 5 & $0(0 \%)$ & $3(0.9 \%)$ & $0(0 \%)$ & $2(0.6 \%)$ & $0(0 \%)$ & $1(0.3 \%)$ & \\
\hline & 6 & $9(8.2 \%)$ & & $9(8.2 \%)$ & & $8(7.3 \%)$ & & \\
\hline & 7 & $2(1.8 \%)$ & & $2(1.8 \%)$ & & $1(0.9 \%)$ & & \\
\hline \multirow{9}{*}{$\begin{array}{l}\text { Distant } \\
\text { metastasis }\end{array}$} & 0 & 17 (15\%) & - & 15 (13.6\%) & - & 14 (13\%) & - & \multirow{9}{*}{$\begin{array}{c}0.50^{* *} \\
1.00^{* * *} \\
0.50^{* * * *}\end{array}$} \\
\hline & 1 & 15 (14\%) & 20 (6.1\%) & 14 (12.7\%) & 19 (5.7\%) & 14 (13\%) & 19 (5.7\%) & \\
\hline & 2 & 16 (15\%) & 24 (7.3\%) & $16(15 \%)$ & 24 (7.2\%) & 16 (14.5\%) & $24(7.2 \%)$ & \\
\hline & 3 & 31 (28\%) & 117 (36\%) & 31 (28\%) & 118 (35\%) & 31 (28\%) & 118 (35\%) & \\
\hline & 4 & 57 (51.8\%) & 124 (37.7\%) & 59 (54\%) & 127 (38\%) & 59 (53.6\%) & 129 (39\%) & \\
\hline & & & 11 (3.3\%) & & & & & \\
\hline & 5 & $4(3.6 \%)$ & $14(4.2 \%)$ & $4(3.6 \%)$ & 12 (3.6\%) & $4(3.6 \%)$ & 13 (3.9\%) & \\
\hline & 6 & 13 (11.8\%) & 19 (5.8\%) & 14 (12.7\%) & 15 (4.5\%) & 13 (12\%) & $14(4.2 \%)$ & \\
\hline & 7 & $8(7.3 \%)$ & & $8(7.3 \%)$ & 18 (5.4\%) & 7 (6.4\%) & 17 (5.1\%) & \\
\hline
\end{tabular}

PET, positron emission tomography; NCCT, non-contrast computed tomography; CECT, contrast-enhanced CT; score 0= no abnormality detected; 1 = focal FDG uptake without CT abnormality; 2 = focal FDG uptake with CT abnormality, favored benign; $3=$ focal FDG uptake with CT abnormality, favored malignant; $4=$ CT abnormality without FDG avidity, favored benign; $5=$ CT abnormality without FDG avidity, favored malignant; $6=$ focal FDG uptake with CT abnormality, indeterminate and $7=$ CT abnormality without FDG avidity, indeterminate $P$ value of **PET/NCCT vs PET/CECT, ***PET/CECT vs PET/NCCT-CECT, ****PET/NCCT vs PET/NCCT-CECT

Table 3. Lesion characterization agreement among different combinations of PET/CT techniques

\begin{tabular}{|c|c|c|c|c|c|c|}
\hline \multirow{2}{*}{ Characterization } & \multicolumn{2}{|l|}{ PET/NCCT vs. PET/CECT } & \multicolumn{2}{l|}{ PET/CECT vs. PET/NC_CECT } & \multicolumn{2}{l|}{ PET/NCCT vs. PET/NC_CECT } \\
\cline { 2 - 7 } & Kappa (SD) & $95 \%$ CI & Kappa (SD) & $95 \%$ CI & Kappa (SD) & $95 \%$ CI \\
\hline Score & $0.941(0.01)$ & $0.913-0.969$ & $0.808(0.02)$ & $0.763-0.853$ & $0.762(0.02)$ & $0.713-0.810$ \\
\hline Definite vs. Indeterminate & $0.864(0.03)$ & $0.799-0.929$ & $1.000(0.00)$ & $1.000-1.000$ & $0.864(0.03)$ & $0.799-0.929$ \\
\hline
\end{tabular}


PET, positron emission tomography; NCCT, non-contrast computed tomography; CECT, contrast-enhanced CT

Table 4. Correlation between potential factors and the possibility of indeterminate lesions detected by each PET/CT technique

\begin{tabular}{|c|c|c|c|c|c|c|}
\hline Factors & Site & Interpreted results & PET/NCCT & $\mathrm{PET} / \mathrm{CECT}$ & PET/NCCT-CECT & $p$-value \\
\hline \multirow{6}{*}{$\begin{array}{l}\text { Age (year) } \\
\text { mean } \pm \text { SD }\end{array}$} & \multirow[t]{2}{*}{ Primary } & Definite & $50.43 \pm 19.07$ & $49.94 \pm 18.81$ & $49.86 \pm 18.62$ & \multirow[t]{2}{*}{$*$} \\
\hline & & Indeterminate & $53.43 \pm 15.49$ & $54.67 \pm 16.58$ & $54.67 \pm 16.58$ & \\
\hline & \multirow[t]{2}{*}{ Node } & Definite & $53.57 \pm 17.27$ & $53.79 \pm 17.03$ & $53.79 \pm 17.03$ & \multirow[t]{2}{*}{$*$} \\
\hline & & Indeterminate & $50.50 \pm 18.64$ & $50.00 \pm 20.68$ & $50.00 \pm 20.68$ & \\
\hline & \multirow[t]{2}{*}{ Metastasis } & Definite & $52.20 \pm 17.65$ & $52.52 \pm 17.18$ & $52.22 \pm 17.32$ & \multirow[t]{2}{*}{$*$} \\
\hline & & Indeterminate & $55.79 \pm 15.70$ & $55.52 \pm 16.18$ & $56.32 \pm 15.63$ & \\
\hline \multirow{6}{*}{$\begin{array}{c}\text { Gender } \\
\% \text { Female }\end{array}$} & Primary & Definite & $60.87 \%$ & $61.22 \%$ & $60.00 \%$ & \multirow[t]{2}{*}{$*$} \\
\hline & & Indeterminate & $57.14 \%$ & $66.67 \%$ & $66.67 \%$ & \\
\hline & Node & Definite & $44.59 \%$ & $46.15 \%$ & $46.15 \%$ & \multirow[t]{2}{*}{$*$} \\
\hline & & Indeterminate & $50.00 \%$ & $50.00 \%$ & $50.00 \%$ & \\
\hline & Metastasis & Definite & $55.00 \%$ & $56.45 \%$ & $57.81 \%$ & \multirow[t]{2}{*}{$*$} \\
\hline & & Indeterminate & $45.45 \%$ & $45.45 \%$ & $41.93 \%$ & \\
\hline \multirow{6}{*}{$\begin{array}{c}\text { BMI }\left(\mathrm{kg} / \mathrm{m}^{2}\right) \\
\text { mean } \pm \mathrm{SD}\end{array}$} & Primary & Definite & $23.28 \pm-3.61$ & $23.32 \pm-3.51$ & $23.36 \pm 3.48$ & \multirow[t]{2}{*}{$*$} \\
\hline & & Indeterminate & $23.83 \pm 5.09$ & $23.75 \pm 5.57$ & $23.75 \pm 5.57$ & \\
\hline & Node & Definite & $23.32 \pm 3.56$ & $23.24 \pm 3.50$ & $23.24 \pm 3.50$ & \multirow[t]{2}{*}{$*$} \\
\hline & & Indeterminate & $23.08 \pm 2.88$ & $23.54 \pm 3.00$ & $23.54 \pm 3.00$ & \\
\hline & Metastasis & Definite & $22.88 \pm 3.58$ & $22.77 \pm 3.34$ & $22.89 \pm 3.50$ & \multirow[t]{2}{*}{$*$} \\
\hline & & Indeterminate & $23.86 \pm 3.93$ & $24.17 \pm 4.16$ & $23.36 \pm 3.48$ & \\
\hline \multirow{6}{*}{$\begin{array}{c}\text { FBS }(\mathrm{mg} / \mathrm{dL}) \\
\text { mean } \pm \text { SD }\end{array}$} & Primary & Definite & $94.93 \pm 16.118$ & $94.35 \pm 15.79$ & $94.74 \pm 15.88$ & \multirow[t]{2}{*}{$*$} \\
\hline & & Indeterminate & $86.00 \pm 11.79$ & $86.17 \pm 12.91$ & $86.17 \pm 12.91$ & \\
\hline & Node & Definite & $95.69 \pm 17.12$ & $95.41 \pm 16.77$ & $95.41 \pm 16.77$ & \multirow[t]{2}{*}{$*$} \\
\hline & & Indeterminate & $87.38 \pm 4.50$ & $88.00 \pm 4.89$ & $88.00 \pm 4.89$ & \\
\hline & Metastasis & Definite & $94.63 \pm 15.07$ & $94.40 \pm 15.07$ & $93.91 \pm 15.09$ & \multirow[t]{2}{*}{$*$} \\
\hline & & Indeterminate & $94.79 \pm 15.10$ & $94.97 \pm 14.88$ & $96.03 \pm 14.71$ & \\
\hline \multirow{6}{*}{$\begin{array}{l}\text { Size }(\mathrm{cm}) \\
\text { mean } \pm \text { SD }\end{array}$} & Primary & Definite & $2.32 \pm 2.26$ & $2.30 \pm 2.25$ & $2.30 \pm 2.25$ & \multirow[t]{2}{*}{$* *$} \\
\hline & & Indeterminate & $1.59 \pm 1.52$ & $1.68 \pm 1.54$ & $1.68 \pm 1.54$ & \\
\hline & Node & Definite & $1.29 \pm 1.05$ & $1.32 \pm 1.05$ & $1.32 \pm 1.05$ & \multirow[t]{2}{*}{ * } \\
\hline & & Indeterminate & $1.21 \pm 0.79$ & $1.09 \pm 0.75$ & $1.09 \pm 0.75$ & \\
\hline & Metastasis & Definite & $1.39 \pm 1.16$ & $1.43 \pm 1.15$ & $1.43 \pm 1.15$ & \multirow[t]{2}{*}{$* * *, * * * *$} \\
\hline & & Indeterminate & $2.05 \pm 1.81$ & $2.26 \pm 2.10$ & $2.26 \pm 2.10$ & \\
\hline
\end{tabular}

PET, positron emission tomography; NCCT, non-contrast computed tomography; CECT, contrast-enhanced CT; BMI, body mass index; FBS, fasting blood sugar, *p $>0.05$ in all techniques, $* *$ p $<0.05$ in NCCT, ***p $<0.05$ in CECT, ****p $<0.05$ in NCCTCECT

Table 5. Positive likelihood ratio for malignancy by score of lesions obtained from each PET/CT technique 


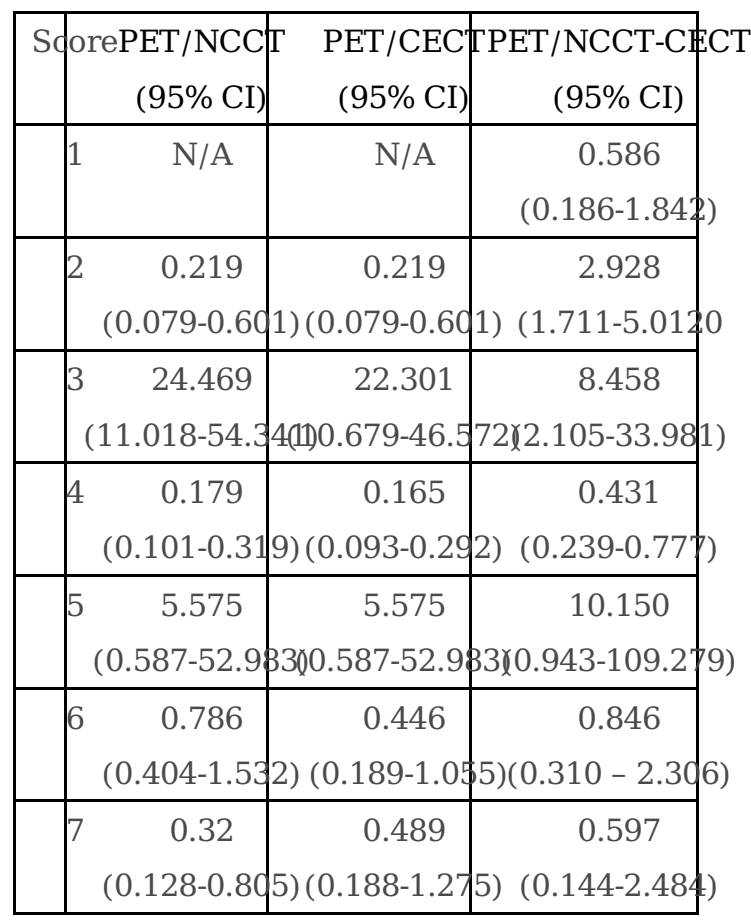

PET, positron emission tomography; NCCT, non-contrast computed tomography; CECT, contrast-enhanced CT; Score $1=$ focal FDG uptake without CT abnormality; 2 = focal FDG uptake with CT abnormality, favoring benign; 3 = focal FDG uptake with CT abnormality, favoring malignant; $4=\mathrm{CT}$ abnormality without FDG avidity, favoring benign; $5=\mathrm{CT}$ abnormality without FDG avidity, favoring malignant; $6=$ focal FDG uptake with CT abnormality, indeterminate; and, $7=\mathrm{CT}$ abnormality without FDG avidity, indeterminate; N/A = not available

Table 6. Correlation between final diagnosis and interpretation obtained from PET/NCCT and PET/CECT 


\begin{tabular}{|c|c|c|c|c|c|c|c|c|c|}
\hline \multirow{2}{*}{$\begin{array}{c}\text { Final } \\
\text { diagnosis }\end{array}$} & & & \multicolumn{6}{|c|}{ PET/CECT } & \multirow[t]{2}{*}{ Total } \\
\hline & & & $\begin{array}{l}\text { FDG +, } \\
\text { CT } \\
\text { benign }\end{array}$ & $\begin{array}{c}\text { FDG +, } \\
\text { CT } \\
\text { malignant }\end{array}$ & $\begin{array}{c}\text { FDG -, } \\
\text { CT } \\
\text { benign }\end{array}$ & $\begin{array}{c}\text { FDG -, } \\
\text { CT } \\
\text { malignant }\end{array}$ & $\begin{array}{c}\text { FDG +, } \\
\text { CT } \\
\text { indeterminate }\end{array}$ & $\begin{array}{c}\text { FDG -, } \\
\text { CT } \\
\text { indeterminate }\end{array}$ & \\
\hline \multirow[t]{7}{*}{ Benign } & \multirow[t]{6}{*}{$\mathrm{PET} / \mathrm{NCCT}$} & $\begin{array}{l}\text { FDG +, CT } \\
\text { benign }\end{array}$ & 34 & 0 & 0 & 0 & 0 & 0 & 34 \\
\hline & & $\begin{array}{l}\text { FDG +, CT } \\
\text { malignant }\end{array}$ & 0 & 6 & 0 & 0 & 0 & 0 & 6 \\
\hline & & $\begin{array}{l}\text { FDG -, CT } \\
\text { benign }\end{array}$ & 0 & 0 & 114 & 0 & 0 & 0 & 114 \\
\hline & & $\begin{array}{l}\text { FDG -, CT } \\
\text { malignant }\end{array}$ & 0 & 0 & 0 & 1 & 0 & 0 & 1 \\
\hline & & $\begin{array}{l}\text { FDG +, } \\
\text { CT } \\
\text { indeterminate }\end{array}$ & 0 & 1 & 0 & 0 & 25 & 0 & 26 \\
\hline & & $\begin{array}{l}\text { FDG -, } \\
\text { CT } \\
\text { indeterminate }\end{array}$ & 0 & 0 & 10 & 0 & 0 & 19 & 29 \\
\hline & \multicolumn{2}{|c|}{ Total } & 34 & 7 & 124 & 1 & 25 & 19 & 210 \\
\hline \multirow[t]{7}{*}{ Malignant } & \multirow[t]{6}{*}{ PET/NCCT } & $\begin{array}{l}\text { FDG +, CT } \\
\text { benign }\end{array}$ & 4 & 0 & 0 & 0 & 0 & 0 & 4 \\
\hline & & $\begin{array}{l}\text { FDG }+, \mathrm{CT} \\
\text { malignant }\end{array}$ & 0 & 79 & 0 & 0 & 0 & 0 & 79 \\
\hline & & $\begin{array}{l}\text { FDG -, CT } \\
\text { benign }\end{array}$ & 0 & 0 & 11 & 0 & 0 & 0 & 11 \\
\hline & & $\begin{array}{l}\text { FDG -, CT } \\
\text { malignant }\end{array}$ & 0 & 0 & 0 & 3 & 0 & 0 & 3 \\
\hline & & $\begin{array}{l}\text { FDG +, } \\
\text { CT } \\
\text { indeterminate }\end{array}$ & 0 & 5 & 0 & 0 & 6 & 0 & 11 \\
\hline & & $\begin{array}{l}\text { FDG -, } \\
\text { CT } \\
\text { indeterminate }\end{array}$ & 0 & 0 & 0 & 0 & 0 & 5 & 5 \\
\hline & \multicolumn{2}{|c|}{ Total } & 4 & 84 & 11 & 3 & 6 & 5 & 113 \\
\hline \multirow[t]{5}{*}{ Total } & \multirow[t]{5}{*}{ PET/NCCT } & $\begin{array}{l}\text { FDG +, CT } \\
\text { benign }\end{array}$ & 38 & 0 & 0 & 0 & 0 & 0 & 38 \\
\hline & & $\begin{array}{l}\text { FDG }+, \text { CT } \\
\text { malignant }\end{array}$ & 0 & 85 & 0 & 0 & 0 & 0 & 85 \\
\hline & & $\begin{array}{l}\text { FDG -, CT } \\
\text { benign }\end{array}$ & 0 & 0 & 125 & 0 & 0 & 0 & 125 \\
\hline & & $\begin{array}{l}\text { FDG -, CT } \\
\text { malignant }\end{array}$ & 0 & 0 & 0 & 4 & 0 & 0 & 4 \\
\hline & & FDG +, & 0 & 6 & 0 & 0 & 31 & 0 & 37 \\
\hline
\end{tabular}




\begin{tabular}{|l|l|l|l|l|l|l|l|} 
& $\begin{array}{l}\text { CT } \\
\text { indeterminate }\end{array}$ & & & & & & \\
\hline & $\begin{array}{l}\text { FDG -, } \\
\text { CT } \\
\text { indeterminate }\end{array}$ & 0 & 0 & 10 & 0 & 0 & 24 \\
\\
\hline Total & $\mathbf{3 8}$ & $\mathbf{9 1}$ & $\mathbf{1 3 5}$ & $\mathbf{4}$ & $\mathbf{3 1}$ & $\mathbf{2 4}$ & $\mathbf{3 2 3}$ \\
\hline
\end{tabular}

PET, positron emission tomography; NCCT, non-contrast computed tomography; CECT, contrast-enhanced CT; FDG +, positive FDG uptake; FDG -, negative FDG uptake

\section{Figures}

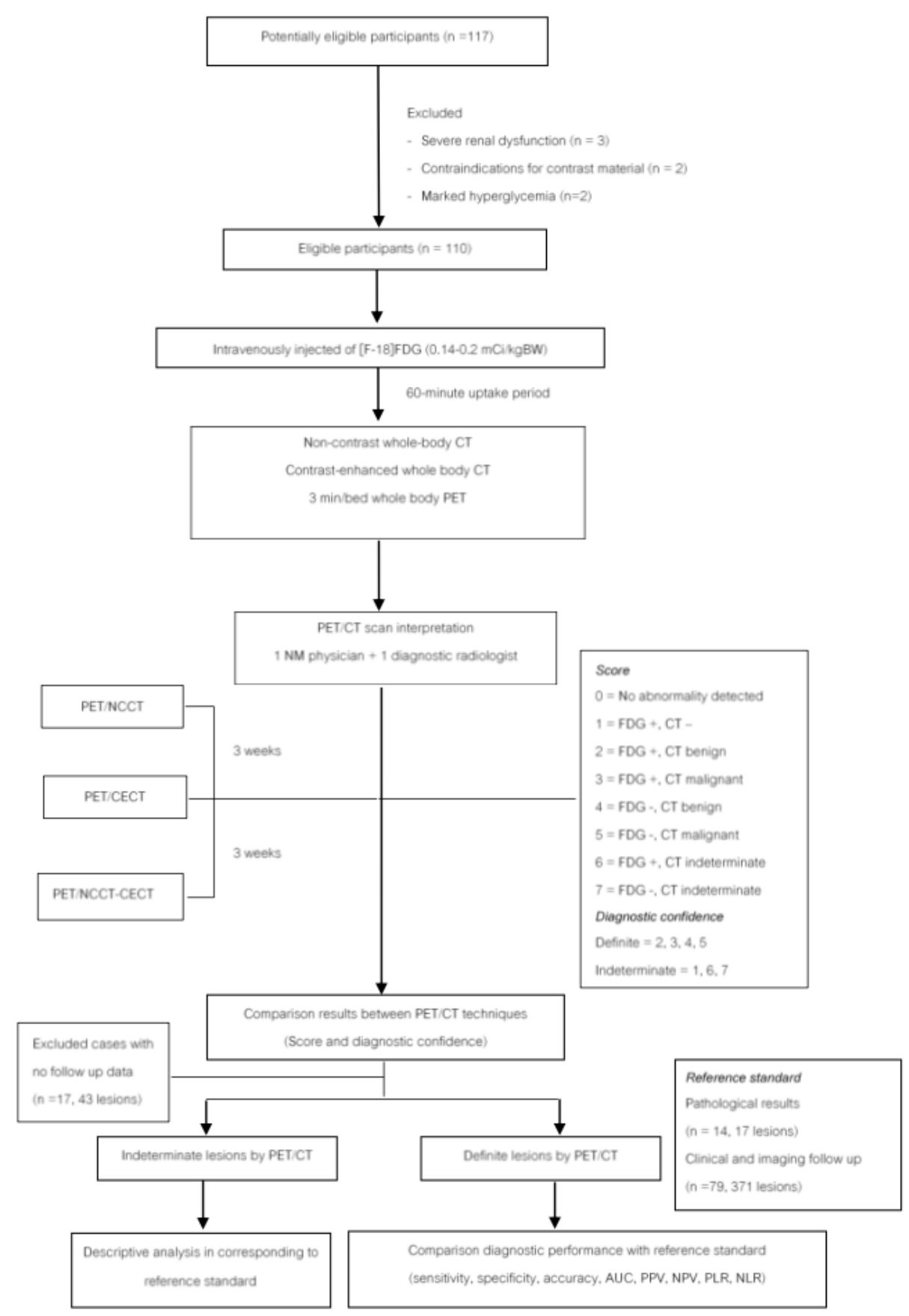




\section{Figure 1}

Flow diagram of patient enrollment and the study protocol

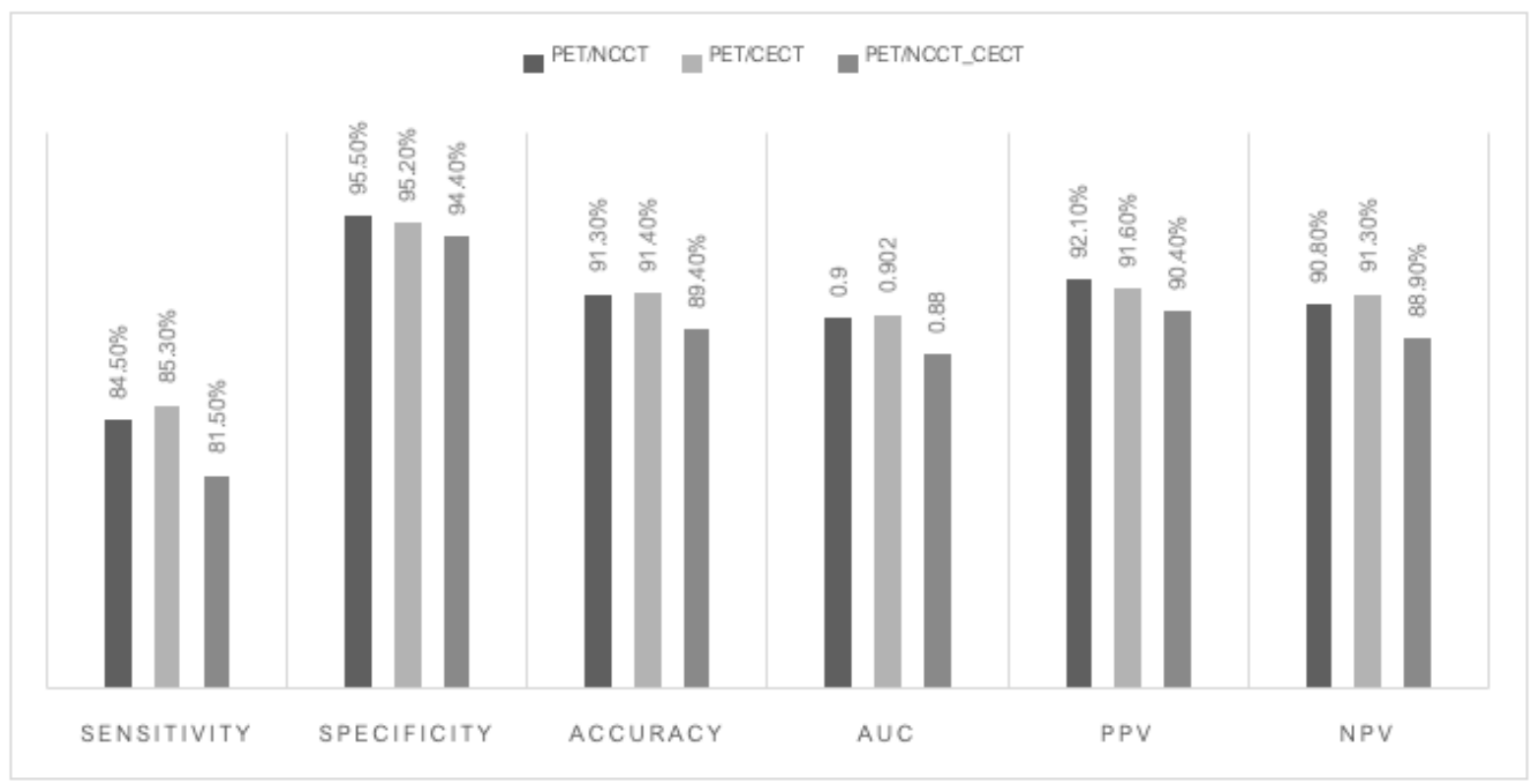

Figure 2

Diagnostic performance among PET/NCCT, PET/CECT, and PET/NCCT-CECT * PET, positron emission tomography; NCCT, non-contrast computed tomography; CECT, contrast-enhanced CT; AUC, area under the curve; PLR, positive likelihood ratio; NLR, negative likelihood ratio; PPV, positive predictive value; NPV, negative predictive value *Indeterminate results by each PET/CT technique were excluded 